\title{
ENSINO E AVALIAÇÃO NO ENSINO SUPERIOR: REFLEXÕES A PARTIR DA PESQUISA REALIZADA NO ÂMBITO DO PROJETO AVENA
}

Domingos Fernandes ${ }^{1}$

RESUMO: O propósito deste artigo foi analisar práticas de ensino e avaliação de docentes de quatro universidades portuguesas, a partir do projeto Avaliação, Ensino e Aprendizagem em Portugal e no Brasil: Realidades e Perspectivas (AVENA). Na chamada fase intensiva do projeto, baseada em observaçóes de aulas e em entrevistas a docentes e estudantes, os dados foram integrados por meio de quatro metanarrativas. A análise mostrou que, em geral, as práticas de ensino e avaliação eram consistentes com uma pedagogia centrada no docente, baseada na exposição das matérias e na avaliação para classificar. Porém, a pesquisa também permitiu identificar práticas de docentes mais baseadas na avaliação para aprender e na distribuição de feedback, em que os estudantes estavam no centro das dinâmicas pedagógicas, participando ativamente na construçáo de sua aprendizagem. $\mathrm{Ou}$ seja, práticas associadas a ambientes pedagógicos que favorecem melhor e mais profunda aprendizagem por parte dos estudantes.

Palavras-chave: Ensino superior. Ensino. Avaliação. Práticas pedagógicas. Práticas curriculares.

${ }^{1}$ Universidade de Lisboa, Instituto de Educação - Lisboa, Portugal.

E-mail: dfernandes@ie.ulisboa.pt

DOI: 10.1590/CC0101-32622016160370 


\title{
Teaching and assessing in higher education: reflections based upon research done within the AVENA project
}

\begin{abstract}
The purpose of this paper was to analyse teaching and assessment practices of teachers belonging to four Portuguese universities, based upon research done within the project Assessment, Teaching, and Learning in Portugal and Brazil: Realities and Perspectives (AVENA). In the so-called intensive phase of the project, based upon class observations and interviews to both teachers and students, data were integrated through four metanarratives. Data analysis showed that, mostly, teaching and assessment practices were consistent with a teachercentered pedagogy, based upon telling and lecturing and in assessment for grading. However, the research also enabled to identify teachers' practices based upon assessment for learning and on the distribution of feedback; students were at the center of the pedagogical processes, being active participants in building their own learning. That is, teaching and assessment practices that were related to pedagogical environments leading to students' better and deeper learning were identified as well.
\end{abstract}

Keywords: Higher education. Teaching. Assessment. Pedagogical practices. Curricular practices.

\section{INTRODUÇÃO}

O presente artigo baseia-se em resultados de uma pesquisa realizada no âmbito do projeto Avaliação, Ensino e Aprendizagem em Portugal e no Brasil: Realidades e Perspectivas (AVENA), que foi financiado em Portugal pela Fundaçáo para a Ciência e Tecnologia (Projeto PTDC/CPE-CED/114318/2009). A pesquisa envolveu 4 universidades portuguesas e 3 universidades brasileiras, 36 pesquisadores seniores e cerca de 20 pesquisadores juniores.

Os dois propósitos fundamentais da pesquisa eram conhecer e compreender práticas de ensino e de avaliação de docentes do ensino superior e percepçóes de docentes e estudantes relativas a uma diversidade de dimensôes daquelas práticas. Fernandes, Rodrigues e Nunes (2012) fazem 
uma descrição detalhada dos fundamentos, do problema, das questóes e da metodologia do projeto AVENA.

Para efeitos deste artigo foram considerados apenas os dados relativos às quatro universidades portuguesas e analisados exclusivamente os dados referentes às práticas docentes nos domínios do ensino e da avaliação. Por meio deste trabalho pretendeu-se descrever, analisar e interpretar práticas de ensino e avaliação dos docentes participantes e produzir reflexóes decorrentes da literatura e dos dados da pesquisa realizada. Assim, não se discutiram outras dimensóes do projeto, nomeadamente a participação dos estudantes em seus processos de aprendizagem, nem percepçôes de estudantes e docentes.

\section{ENQUADRAMENTO TEÓRICO E CONCEITUAL}

O desenvolvimento do Espaço Europeu do Ensino Superior (EEES) tem sido baseado na Declaração de Bolonha, de 1999, também designada como Processo de Bolonha, que veio questionar as instaladas rotinas das instituiçôes europeias de ensino superior através de uma agenda pedagógica que, para além das controvérsias, conseguiu conquistar a simpatia de largos setores do mundo acadêmico e da sociedade em geral (FERNANDES, 2014). De certo modo, os dirigentes políticos decidiram corroborar ideias há muito defendidas na literatura e nos meios acadêmicos para que a melhoria da aprendizagem, da avaliaçáo pedagógica e do ensino passassem a estar no centro das preocupaçóes das instituiçóes. Assim, a Pedagogia passou a estar na ordem do dia da vida universitária. Consequentemente, proliferaram todos os tipos de iniciativas que deram origem a um significativo número de publicaçóes em que, nomeadamente, se referia como necessário:

- apostar na articulação e/ou integração dos processos de aprendizagem, avaliação e ensino;

- criar condiçóes para melhorar conhecimentos e capacidades dos docentes em domínios tais como a aprendizagem, a avaliação e o ensino;

- $\quad$ promover a autonomia e a participação dos estudantes nas açóes relacionadas ao desenvolvimento do currículo; 
- incentivar a interação social entre os estudantes e entre estes e os docentes;

- $\quad$ reconhecer a possibilidade de melhorar as práticas curriculares dos docentes (BRYAN; CLEGG, 2006; FALCHICOV, 2005).

Esse ímpeto reformista orientado para a transformação e a melhoria da chamada Pedagogia do/no Ensino Superior não foi, nem está sendo, desenvolvido independentemente das contradiçóes que têm afetado as políticas públicas para o ensino superior, marcadas por tendências como:

- $\quad$ a aproximação às lógicas de mercado por meio de estratégias de natureza mercantil;

- a fusão entre instituiçóes com vistas à internacionalização e ao aumento de sua capacidade competitiva, muitas vezes medida por rankings internacionais;

- a utilização de processos de avaliação indutores de mudanças importantes no trabalho acadêmico.

Essas tendências condicionam a vida acadêmica e profissional dos docentes universitários, cada vez mais sob a mira de processos de regulação que ameaçam a autonomia e a liberdade acadêmica (ESCUDERO, 2012). Apesar disso, para esse autor, a polêmica em torno do chamado Processo de Bolonha está mais nas perspectivas técnico-burocráticas e mercantis que o têm influenciado do que na agenda pedagógica proposta em seu âmbito. Na verdade, essa agenda propóe mudanças há muito reclamadas e necessárias para melhorar a qualidade da formação dos estudantes.

Uma análise de literatura publicada em revistas internacionais de referência na primeira década deste século permitiu identificar práticas de ensino e de avaliação normalmente associadas à melhoria da aprendizagem. Num conjunto de pesquisas (KLENOWSKI; ASKEW; CARNELL, 2006; MILLER, 2009) os docentes utilizavam uma variedade de tarefas de avaliação, em geral de duas a três, sobre as quais os estudantes trabalhavam num clima em que a interação com os colegas e os 
docentes, a distribuição de feedback, a partilha de reflexões e a utilização de dinâmicas diversificadas de ensino e de aprendizagem eram características marcantes. Noutros estudos (DANCER; KAMVOUNIAS, 2005; NICOL, 2009; POON et al., 2009) verificou-se a participação sistemática dos estudantes em ações diretamente relacionadas à avaliação das aprendizagens de acordo com dinâmicas tais como:

- trabalho colaborativo em pequenos grupos, analisando tarefas, planejando e gerindo o tempo, distribuindo e partilhando feedback;

- $\quad$ envolvimento na discussão, na definição e na clarificação de critérios de avaliaçáo relativos às tarefas que lhes eram propostas;

- $\quad$ participação em processos de autoavaliação e de avaliação entre pares.

No que se refere à natureza e à utilização do feedback, a literatura consultada mostrou que, em geral, o feedback era criterial, escrito ou oral, sendo distribuído pelos docentes com o propósito de informar, corrigir, motivar, orientar e atribuir classificaçóes (MACMILLAN; MCLEAN, 2005; TROTTER, 2006). Os estudantes também eram envolvidos na distribuição de feedback aos colegas. Para tal, usavam instrumentos como listas de verificação, rubricas de avaliação ou tabelas que descreviam diferentes níveis de desempenho para determinada tarefa (WALKER; WARHURST, 2000; WANOUS; PROCTER; MURSHIDB, 2009).

A distribuição de feedback tem sido particularmente referida na literatura, uma vez que é reconhecido o seu papel no desenvolvimento dos processos mais complexos de pensamento dos estudantes, bem como na regulação e na autorregulação das suas aprendizagens (ALBERTINO; DE SOUSA, 2004; ANDRADE; DU, 2007; KLENOWSKI; ASKEW; CARNELL, 2006; MACMILLAN; MCLEAN, 2005). A participação dos estudantes na definição e na discussão dos critérios que eram utilizados para avaliar o seu trabalho foi igualmente considerada como estando associada à melhoria de sua aprendizagem. $\mathrm{Na}$ verdade, ao tornarem-se mais conscientes e interiorizarem tais critérios, os estudantes ficavam em melhores condiçóes para avaliar o seu trabalho e o dos colegas e para 
se concentrar no que tinham que aprender; desse modo, os estudantes desenvolviam suas capacidades metacognitivas, de autoavaliação e de avaliação entre pares e de regulação e autorregulação de sua aprendizagem (KLENOWSKI; ASKEW; CARNELL, 2006; MOK et al., 2006; PRICE; O'DONOVAN, 2006).

Em suma, abundam na literatura estudos com sólidas bases empíricas que nos indicam a real possibilidade de melhorar significativamente o conteúdo e a forma do que os estudantes do ensino superior aprendem. As práticas curriculares, em geral, e as práticas de ensino e de avaliação dos docentes, em particular, são elementos fundamentais para que os estudantes aprendam mais e melhor, com compreensão e profundidade.

\section{MÉTODO}

A pesquisa das práticas de ensino e avaliação dos docentes ocorreu na chamada fase intensiva do estudo, baseada em observaçóes de aulas, entrevistas profundas com os docentes e entrevistas com grupos focados de estudantes. $\mathrm{Na}$ fase extensiva, que não foi tratada no âmbito deste artigo, administrou-se um questionário de 45 itens a docentes e estudantes para investigar sua percepção das dimensões da aprendizagem, do ensino e da avaliação. Para uma consulta aprofundada sobre a pesquisa do projeto AVENA nessa fase, consulte, por exemplo, Barreira et al. (2014) e Fernandes (2015).

Da fase intensiva do estudo, realizada nas 4 universidades portuguesas, participaram voluntariamente 35 docentes e 168 estudantes. Observaram-se aulas de natureza prática ou teórico-prática de disciplinas consideradas estruturantes de cursos de licenciatura em cada um dos seguintes domínios do conhecimento: Ciências Sociais, Artes e Humanidades, Ciências da Saúde e Ciências e Tecnologias. Para cada um desses domínios do conhecimento foram observadas, em cada universidade, as aulas de dois docentes, cada um dos quais responsável por uma disciplina. Uma vez que cada docente foi observado durante cerca de 20 horas, realizaram-se cerca de 160 horas de observação de aulas de disciplinas de cada domínio do conhecimento (40 horas por universidade). Assim, no 
total, foram observadas cerca de 640 horas de aulas de todos os domínios do conhecimento. Além disso, realizaram-se entrevistas profundas com cada um dos docentes participantes e entrevistas com grupos focados de três a cinco estudantes cada um.

O processo de integração e transformação de dados, inspirado nas recomendaçóes de Wolcott (1994), foi baseado na produção sucessiva de narrativas orientada pelas dimensóes constantes numa matriz de investigação da pesquisa. Assim, para a avaliação foram consideradas dimensóes como:

- $\quad$ tarefas de avaliação mais utilizadas;

- natureza, frequência e distribuição de feedback;

- $\quad$ papel predominante dos docentes e dos estudantes no processo de avaliação.

Para o ensino foram utilizadas, entre outras, as seguintes dimensóes:

- $\quad$ recursos e materiais utilizados;

- $\quad$ dinâmicas de sala de aula;

- $\quad$ gestão do tempo e estruturação da aula.

A matriz foi fundamental para garantir níveis aceitáveis de consistência entre as narrativas produzidas pelos diferentes pesquisadores. Além do mais, à medida que eram construídas, as narrativas circulavam, para análise e clarificação, pelos quatro grupos de pesquisa das universidades participantes.

Para cada docente foram inicialmente produzidas três narrativas: uma baseada na observação das aulas, outra na entrevista profunda com o docente e outra na entrevista com um grupo focado de estudantes. Através de um processo de integração, as três narrativas davam origem a uma só, referente às práticas de ensino e avaliaçáo de cada docente. Assim, em cada universidade e para cada domínio do conhecimento foram produzidas duas narrativas referentes às práticas dos dois docentes que lecionavam disciplinas nesse domínio. Prosseguindo a integração dos 
dados, essas duas narrativas deram origem a uma só narrativa integrada dos docentes A e B. Assim, em cada universidade foram produzidas quatro narrativas integradas, uma para cada domínio do conhecimento. Finalmente, a partir de cada grupo de quatro narrativas foi construída uma metanarrativa que integrava as práticas dos oito docentes de cada domínio do conhecimento. Cada metanarrativa foi construída de forma interativa entre os diferentes pesquisadores, tendo por base a matriz de investigação e as narrativas integradas. Tendo em conta que todas as narrativas foram devolvidas aos participantes para análise e validação, há razóes para crer que, no mínimo, os seus níveis de consistência e credibilidade são aceitáveis.

O processo de análise, integração e transformação de dados não foi algorítmico nem obedeceu a qualquer sistema clássico de análise de conteúdo. Evitou-se uma abordagem excessivamente analítica, desenvolvendo-se um trabalho mais artesanal na identificação de padrōes, regularidades, discrepâncias assinaláveis, situaçóes singulares e seguindose uma perspectiva indutiva da organização e análise dos dados.

Em suma, a produção das quatro metanarrativas criou condições para que se pudesse descrever, analisar e interpretar as práticas pedagógicas de avaliação e ensino dos docentes participantes.

\section{APRESENTAÇÃO E DISCUSSÃO DE RESULTADOS}

A seguir, apresentam-se e discutem-se alguns dos resultados considerados mais relevantes no domínio das práticas pedagógicas de ensino e de avaliação dos 35 docentes envolvidos na pesquisa. Para tal, procedeu-se à análise das metanarrativas construídas para cada domínio do conhecimento na fase intensiva da pesquisa.

De modo geral, a estrutura das aulas, as dinâmicas de ensino e de trabalho, a gestão do tempo e dos espaços, o papel dos estudantes e dos docentes e a participaçáo dos estudantes nas atividades das aulas variaram sensivelmente de docente para docente. $\mathrm{Na}$ verdade, constatou-se que cada docente desenvolvia o currículo obedecendo a diferentes perspectivas de organização do trabalho pedagógico baseadas nas suas visóes, experiências e concepçóes do que é ensinar e do que é aprender. 
Na maioria dos casos, os docentes estavam no centro do processo pedagógico, paradigma da Pedagogia Centrada no Docente, fazendo suas preleçóes enquanto os estudantes se limitavam a tomar notas e/ou a ouvir o que era dito. $\mathrm{O}$ ensino magistral era a prática dominante nas aulas observadas, todas elas de natureza teórico-prática ou prática.

Um número reduzido de docentes, cerca de um terço, desenvolveu práticas de ensino mais consentâneas com o chamado paradigma da Pedagogia Centrada no Estudante. Em suas aulas, os estudantes participaram do desenvolvimento das atividades, as tarefas eram diversificadas, discutia-se e sintetizava-se o que se ia fazendo e utilizaram-se diferentes dinâmicas de trabalho.

É importante referir que, em algumas aulas nas quais os docentes ocupavam o centro dos processos pedagógicos, falando quase o tempo todo enquanto os estudantes ouviam e/ou tomavam notas, foi possível verificar que os estudantes se mobilizavam e motivavam para aprender, fazendo referências muito positivas ao trabalho dos docentes. Por outro lado, em algumas aulas em que os estudantes eram supostamente mais autônomos e mais chamados a participar e interagir com os colegas e os docentes, constatou-se que o ambiente pedagógico não era propriamente o mais favorável à aprendizagem. Na verdade, por uma diversidade de razóes (natureza das tarefas, gestão do tempo, natureza do feedback), alguns estudantes não se sentiam envolvidos nas dinâmicas de aprendizagem pretendidas. Essas constataçóes ilustram a natureza complexa dos processos de ensino e dos ambientes pedagógicos nas salas de aula. Por isso mesmo, não são facilmente enquadráveis em ideias pré-concebidas acerca do que, supostamente, é um bom ensino ou um bom ambiente pedagógico.

A estrutura das aulas era, em geral, pouco elaborada e incluía uma breve apresentação do assunto, com ou sem referência ao que tinha sido tratado na aula anterior, seguida da preleçáo do docente durante a maior parte do tempo. Nesses casos, a organização e a dinâmica de trabalho se reduziam à fala do docente, por vezes brilhante e eloquente, dirigida ao grande grupo de estudantes, que se limitavam a ouvir e a tomar notas. As reaçóes dos estudantes a essa abordagem variaram entre a insatisfação, a indiferença, a satisfação e a resignação. Na verdade, os alunos tiveram dificuldade em expressar qualquer pensamento sustentado sobre o que poderiam ser a estrutura e a organização pedagógica das aulas para que pudessem aprender com mais compreensão e profundidade. 
Num número reduzido de casos, a estrutura e a organização das aulas foram mais sofisticadas, geradoras de dinâmicas de trabalho participativas e diversificadas. De fato, as aulas de alguns docentes eram estruturadas em momentos distintos, promovendo a participação dos estudantes, a distribuição do feedback, o uso de diferentes dinâmicas de trabalho e o estímulo à interação entre todos os participantes. Essas aulas incluíam momentos como:

- uma síntese da aula anterior;

- uma introdução feita pelo docente para fundamentar o trabalho a ser desenvolvido;

- uma fase de trabalho autônomo dos estudantes sobre uma tarefa (em pequenos grupos, em pares ou individualmente), podendo contar com o apoio do docente;

- uma síntese do trabalho realizado;

- uma fase de trabalho autônomo dos estudantes sobre a mesma ou outra tarefa, tal como indicado em (c);

- $\quad$ um ponto de situação final acerca do trabalho desenvolvido.

Noutros casos, eram contemplados menos momentos (três ou quatro), mas obedecida a mesma lógica de incentivar os estudantes a participarem mais ativamente dos processos pedagógicos conducentes à sua aprendizagem. Nas aulas que se estruturavam dessa forma, a articulação entre a avaliação, o ensino e a aprendizagem surgia naturalmente, pois cada tarefa era um meio através do qual se aprendia, se ensinava e se avaliava.

As práticas de avaliação, sobretudo no âmbito da avaliação para a aprendizagem, são indissociáveis da distribuição de feedback, processo incontornável para a regulação da aprendizagem. A pesquisa mostrou que, nas aulas em que os estudantes tinham papel preponderante, of fee$d b a c k$ era distribuído de forma sistemática sob várias formas, tinha como finalidade apoiar, orientar e regular a aprendizagem e estava bem articulado com a avaliação, o ensino e a aprendizagem. Além do mais, as rotinas pedagógicas das aulas incluíam momentos de autoavaliação e de 
avaliação entre pares, promovendo oportunidades para os estudantes refletirem sobre o seu trabalho e o dos colegas. Tanto os estudantes quanto os docentes valorizaram o papel relevante desempenhado pelo feedback na melhoria dos processos de aprendizagem.

Quando os docentes ocupavam o centro da ação pedagógica, a estrutura do ensino, a participaçáo dos estudantes — reduzida ou mesmo ausente - e a predominância de avaliação orientada para as classificaçóes não favoreciam a prática do feedback com o propósito de apoiar a aprendizagem, ajudando os estudantes a regularem e orientarem o seu trabalho. Assim, a única utilização dada à avaliação parecia ser a certificação da aprendizagem supostamente desenvolvida pelos estudantes. De modo geral, os estudantes mostraram-se bastante críticos e até desapontados com os propósitos e as utilizaçôes da avaliação, não identificando nesse processo uma forma de ajudá-los a aprender. Para a grande maioria deles, a avaliação apenas servia para classificá-los e não traduzia com rigor o que sabiam e eram capazes de fazer. Os docentes, por sua vez, aparentemente mais otimistas, consideraram que utilizavam a avaliação para "detectar dificuldades", "adaptar o ensino" e "aferir conhecimentos". Porém, nas observaçôes efetuadas não foi possível identificar essas utilizações.

Em resumo, pode-se dizer que, em geral, predominou uma matriz pedagógica em que os docentes tinham protagonismo, com os estudantes essencialmente passivos, limitando-se a tomar notas do que ouviam - um ensino de natureza magistral e uma avaliaçáo essencialmente orientada para classificar e/ou certificar. Porém, ainda que num número reduzido de docentes (cerca de 30\%), foi possível identificar aulas em que os estudantes tinham níveis de protagonismo mais ou menos elevados, participando ativamente dos processos pedagógicos de aprendizagem, avaliação e ensino. Nesses casos, a avaliaçáo era essencialmente contínua, formativa e orientada para apoiar o ensino e a aprendizagem.

\section{CONCLUSÕES E REFLEXÕES FINAIS}

O propósito do projeto AVENA era descrever e procurar compreender os modos como os docentes organizavam suas práticas, tendo em conta uma diversidade de dimensôes, tais como: a estrutura e a dinâ- 
mica das aulas, a natureza do feedback, os propósitos e as utilizações das avaliaçóes e os papéis de docentes e estudantes.

Uma das conclusóes desta pesquisa corroborou o que vinha sendo sinalizado na literatura (ALBERTINO; DE SOUSA, 2004; KLENOWSKI; ASKEW; CARNELL, 2006), referindo que o trabalho pedagógico realizado no contexto das salas de aula pode ser decisivo para que os estudantes aprendam mais e melhor. Para tal, eles devem ser ativos protagonistas nas dinâmicas de trabalho e, consequentemente, na resolução de uma diversidade de tarefas. $\mathrm{O}$ trabalho pedagógico pode ser gerador de ambientes em que o ensino, a avaliaçáo e a aprendizagem estejam fortemente relacionados e organizados para apoiar e melhorar a qualidade do que se aprende.

Tal como nos é referido por Escudero (2012), esta pesquisa também evidenciou que os docentes podem desenvolver práticas de ensino e de avaliaçáo inovadoras, ainda que os contextos institucionais e/ou político-sociais não o favoreçam. Ou seja, os docentes podem inovar em dimensões como a organização e a estruturação do ensino, a participação dos estudantes, a seleção de tarefas, a natureza da avaliação e do feedback e as dinâmicas de trabalho nas salas de aula. $\mathrm{O}$ trabalho empírico permitiu identificar um largo espectro de práticas docentes, que inclui desde as que situam o docente no centro dos processos de educação e formação até aquelas que garantem aos estudantes um destacado protagonismo na construção de sua aprendizagem. Se quisermos, num extremo temos uma Pedagogia Centrada no Docente e, no outro, uma Pedagogia Centrada no Estudante. No primeiro caso, claramente preponderante, predominaram as práticas de ensino mais técnicas e estandardizadas, a fala do docente, a passividade dos estudantes e a avaliação orientada para as classificaçóes. No segundo, minoritário, prevaleceram a participação dos estudantes, as práticas pedagógicas mais reflexivas e críticas e o ensino e a avaliação mais inovadores. Independentemente das suas práticas, os docentes pareceram agir mais baseados na intuição do que no conhecimento pedagógico deliberadamente mobilizado. Essa constatação suscita, naturalmente, questóes diversas no domínio da formação pedagógica e do desenvolvimento profissional dos docentes do ensino superior.

Os resultados desta pesquisa são consistentes com os obtidos por Berg, Admiraal e Pilot (2006) no que se refere aos benefícios que os estudantes obtêm com sua participação em processos de autoavaliação e de 
avaliação entre pares, porque se concentram melhor no que têm de aprender, melhorando seus níveis de atenção e motivação. Da mesma forma, tal como verificado por Nicol (2009), os estudantes valorizaram os momentos de reflexão e de balanço proporcionados por aqueles tipos de avaliação.

A análise das metanarrativas também sugeriu que a articulação entre a natureza das tarefas, a estrutura das aulas e as dinâmicas de trabalho cria contextos mais favoráveis à aprendizagem dos estudantes. A relação pedagógica, que passa pela distribuição sistemática de feedback de qualidade, foi identificada como um processo que permitiu enquadrar os estudantes, induzindo sentimentos de segurança, confiança e autoestima, fundamentais para o desenvolvimento de sua autonomia e de seu envolvimento nos processo de aprendizagem. A literatura revista permitiu constatar esses tipos de relaçóes, que devem ser estudadas e compreendidas com mais profundidade (FERNANDES; FIALHO, 2012).

A distribuição de feedback foi muito valorizada pelos estudantes em razão das orientaçóes que lhes proporcionava para clarificar o que tinham que aprender, a situação em que se encontravam e os esforços que deveriam fazer para regular e autorregular sua aprendizagem. Tal como evidenciado na pesquisa de Trotter (2006), verificou-se que, em geral, o feedback era de natureza criterial e utilizado com propósitos formativos, mas também classificatórios.

Esta pesquisa mostrou que, em geral, as práticas docentes ainda estão longe daquelas recomendadas pela literatura e pela agenda pedagógica decorrente do Processo de Bolonha. Ensinar é um processo ainda associado à ideia de "dizer", e a avaliação diz respeito aos docentes, significando, em última análise, classificar. Ou seja, a Pedagogia Centrada no Docente continua a predominar nos processos universitários de educação e formação.

Porém, independentemente de outras conclusões que puderam ser inferidas desta pesquisa, identificou-se um conjunto de caraterísticas que, de acordo com os dados obtidos e a literatura revista, está associado à criação de ambientes pedagógicos que favorecem a aprendizagem. Refiram-se, por exemplo, as seguintes:

- aulas com estruturas bem definidas e indutoras da participação ativa dos estudantes; 
- criteriosa seleção de uma diversidade de tarefas para aprender, ensinar e avaliar, que devem ser trabalhadas por meio de diferentes dinâmicas de sala de aula;

- distribuição sistemática e deliberada de feedback tão cedo quanto possível após a realização das tarefas pelos alunos;

- $\quad$ utilização da avaliação formativa para apoiar e melhorar os processos de ensino e aprendizagem;

- $\quad$ elaboraçáo de pontos de situaçáo acerca da aprendizagem realizada.

Essas abordagens foram utilizadas por docentes de quatro domínios do conhecimento, mostrando que é possível desenvolver práticas curriculares mais consentâneas com a melhoria dos processos de educação e formação dos jovens universitários.

\section{REFERÊNCIAS}

ALBERTINO, F.M.F.; DE SOUSA, N.A. Avaliação da aprendizagem: o portfólio como auxiliar na construção de um profissional reflexivo. Estudos em Avaliação Educacional, v. 29, p. 169-189, jan./jun. 2004.

ANDRADE, H.; DU, Y. Student responses to criteria-referenced selfassessment. Assessment and Evaluation in Higher Education, v. 32, n. 2, p. 159-181, abr. 2007.

BARREIRA, C.; BIDARRA, M.G.; VAZ-REBELO, P.; MONTEIRO, F.; ALFERES, V. Percepçóes de docentes e estudantes de universidades portuguesas sobre ensino, aprendizagem e avaliação. In: FERNANDES, D.; BORRALHO, A.; BARREIRA, C.; MONTEIRO, A.; CATANI, D.; CUNHA, E.; ALVES, P. (Orgs.). Avaliação, ensino e aprendizagens em Portugal e no Brasil: realidades e perspectivas. Lisboa: Educa, 2014. p. 309-326. v. 1.

BERG, I.; ADMIRAAL, W.; PILOT, A. Design principles and outcomes of peer assessment in higher education. Studies in Higher Education, v. 31, n. 3, p. 341-356, 2006.

BRYAN, C.; CLEGG, K. Innovative assessment in higher education. New York: Taylor and Francis, 2006. 
DANCER, D.; KAMVOUNIAS, P. Student involvement in assessment: a project designed to assess class participation fairly and reliably. Assessment and Evaluation in Higher Education, v. 30, n. 4, 445-454, ago. 2005.

ESCUDERO, J.M. Claros y oscuros del Espacio Europeo de Educación Superior como innovación educativa. In: LEITE, C.; ZABALZA, M. (Cords.). Ensino superior: inovação e qualidade na docência. Porto: Centro de Investigação e Intervençáo Educativas da Faculdade de Psicologia e de Ciências da Educação da Universidade do Porto, 2012. p. 5-34.

FALCHIKOV, N. Improving assessment through student involvement: practical solutions for aiding learning in higher and further education. New York: Routledge, 2005.

FERNANDES, D. Práticas de ensino e de avaliação de docentes de quatro universidades portuguesas. In: FERNANDES, D.; BORRALHO, A.; BARREIRA, C.; MONTEIRO, A.; CATANI, D.; CUNHA, E.; ALVES, P. (Orgs.). Avaliação, ensino e aprendizagens em Portugal e no Brasil: realidades e perspectivas. Lisboa: Educa, 2014. p. 97-135, v. 1.

FERNANDES, D. Pesquisa de percepções e práticas de avaliação no ensino universitário português. Estudos em Avaliação Educacional, v. 26, n. 63, p. 596629, set./dez. 2015.

FERNANDES, D.; FIALHO, N. Dez anos de práticas de avaliação das aprendizagens no Ensino Superior: uma síntese da literatura (2000-2009). In: LEITE, C.; ZABALZA, M. (Cords.). Ensino superior: inovação e qualidade na docência. Porto: Centro de Investigação e Intervenção Educativas da Faculdade de Psicologia e de Ciências da Educaçáo da Universidade do Porto, 2012. p. 3693-3707.

FERNANDES, D.; RODRIGUES, P.; NUNES, C. Uma investigação em ensino, avaliação e aprendizagens no ensino superior. In: LEITE, C.; ZABALZA, M. (Cords.). Ensino superior: inovação e qualidade na docência. Porto: Centro de Investigação e Intervenção Educativas da Faculdade de Psicologia e de Ciências da Educação da Universidade do Porto, 2012. p. 932-944.

KLENOWSKI, V.; ASKEW, S.; CARNELL, E. Portfolios for learning, assessment and professional development in higher education. Assessment and Evaluation in Higher Education, v. 31, n. 3, p. 267-286, 2006.

MACMILLAN, J.; MCLEAN, M.J. Making first-year tutorials count: operationalizing the assessment-learning connection. Active Learning in Higher Education, v. 6, n. 2, p. 94-105, jul. 2005. 
MILLER, T. Formative computer-based assessment in higher education: the effectiveness of feedback in supporting student learning. Assessment and Evaluation in Higher Education, v. 34, n. 2, p. 181-192, 2009.

MOK, M.M.C.; LUNG, C.L.; CHENG, D.P.W.; CHEUNG, R.H.P.; NG, M.L. Self-assessment in higher education: experience in using a metacognitive approach in five case studies. Assessment and Evaluation in Higher Education, v. 31, n. 4, p. 415-433, 2006.

NICOL, D. Assessment for learner self-regulation: enhancing achievement in first year using learning technologies. Assessment and Evaluation in Higher Education, v. 34 n. 3, p. 335-352, 2009.

POON, W.Y.; MCNAUGHT, C.; LAM, P.; KWAN, H.S. Improving assessment methods in university science education with negotiated self- and peer-assessment. Assessment and Evaluation in Higher Education, v. 16, n. 3, p. 331-346, 2009.

PRICE, M.; O'DONOVAN, B. Improving performance trough enhancing student understanding of criteria and feedback. In: BRYAN, C.; CLEGG, K. (Orgs.). Innovative assessment in higher education. New York: Taylor and Francis Group, 2006. p. 100-109.

TROTTER, E. Student perceptions of continuous summative assessment. Assessment and Evaluation in Higher Education, v. 31, n. 5, p. 505-521, 2006.

WALKER, M.; WARHURST, C. In most classes you sit around very quietly at a table and get lectured at...' debates, assessment and student learning. Teaching in Higher Education, v. 5, n. 1, p. 33-49, 2000.

WANOUS, M.; PROCTER, B.; MURSHIDB, K. Assessment for learner self evaluation: enhancing achievement in first year using learning technologies. European Journal of Engineering Education, v. 34, n. 1, p. 77-85, 2009.

WOLCOTT, H. Transforming qualitative data: description, analysis, and interpretation. London: Sage, 1994.

Recebido em 07 de abril de 2016

Aceito em 22 de agosto de 2016. 